\title{
A SURFACE RELIEF METER BASED ON TRINOCULAR VISION
}

\author{
V G S Ernst*, P W Sablik**, J Balendonck**. Z Houkes*, P P L Regtien* \\ * University of Twente, The Netherlands \\ ** Institute of Agricultural and Environmental Engineering (IMAG-DLO), The Netherlands
}

\section{INTRODUCTION}

Many soil properties, being influenced by tillage, appear to be related to soil surface roughness. Changes in surface roughness affect the exposed surface area, air currents, air radiation which indirectly influence the moisture, temperature and aeration of the soil. Also, the surface movement and storage of water is related to the roughness of configuration of the soil surface. Therefore, measurement of soil surface roughness and correlation of roughness with other soil properties would provide a fast, one-measurement method of quantitatively measuring the effect of tillage on crop production-related soil properties.

Much research effort has been put into the development of relief measurements. Kuipers [1] developed the first relief meter for agricultural application. His system is based on a frame with a number of extensible rods, which rest on the surface. Height information is obtained from the extended length of these rods. Later, a non-contact profile meter is developed by Römkens et al [2]. Their system uses an infra-red LED and a photo transistor. It is tried to keep a rod, with the LED and photo transistor connected to it, at a constant distance from the soil, thus obtaining height information. Later Römkens et al [3] replaced the LED and photo transistor by a LASER and PIN diode.

All of these methods suffer from one or more of the following shortcomings:

- they are not easy manageable and operable by one person,

- they are wasteful of energy,

- it takes a long time to record a surface unit,

- they have a small measuring surface,

- they have a low height accuracy and a low spatial resolution.

In order to overcome these deficiencies, a different approach based on trinocular computer vision is used to develop a relief meter.

\section{TRINOCULAR VISION}

In binocular vision, two cameras are used. If both cameras observe the same scene, this scene is depicted at both CCD chips. Because the two cameras have different geometric positions, the depiction of the scene at one CCD chip will (slightly) differ from the depiction at the other CCD chip. This difference can be used to reconstruct the scene (Smith [4]).

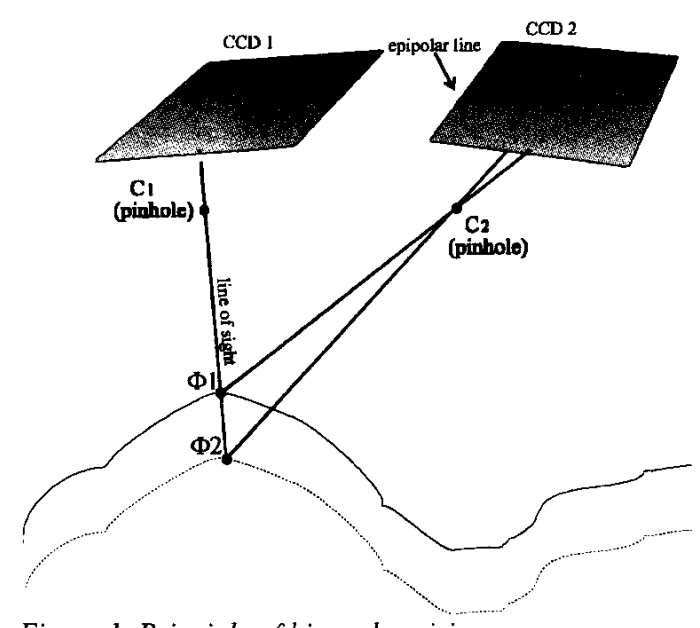

Figure 1. Principle of binocular vision

As depicted by Figure 1, one camera is not sufficient to reconstruct the position of a scene point because each point lying on a line of sight will be depicted at the same point $P_{1}$ on the CCD chip. Herein it is assumed, that the depiction takes place by the so-called pinhole model (De Graaff [5]). In this model, only the rays of light passing through one point (the pinhole) are depicted on the CCD chip.

A second camera can be used to reconstruct scene points. Once it is known that $P_{1}$ and $P_{2}$ are corresponding points, it is known that $\Phi_{1}$ is the true scene point $\left(\boldsymbol{\Phi}_{1}\right.$ is the point of intersection of the two lines of sight). Similarly, once it is known that $P_{1}$ and $P_{3}$ are corresponding image points, it is known that $\Phi_{2}$ is the true scene point. For each image point in the first image, there exists only one image point in the second image that corresponds to this image point. The search for these corresponding image points is called the correspondence problem. This search for corresponding image points is not done for all pixels in the image, but only for a limited set of points called feature points. Feature points can, for example, represent changes in grey value (Grimson [6]). Fortunately, the search for corresponding points is not arbitrary. Once a point $P_{1}$ from the first CCD chip (CCD 1) is selected, it is known that the corresponding scene point should lie on the line of sight of this point. Therefore, the corresponding point in the second CCD chip (CCD 2) must lie on the depiction of this line at CCD 2. This 
line is called the epipolar line. Only points on this line, matching with the corresponding point on $\operatorname{CCD~} 1$, are allowed. In binocular vision there is still a quite large probability of matching the wrong points.

To overcome this problem, a third camera can be added. The principle of operation of trinocular vision (Yachida et al [7]) is presented in Figure 2.

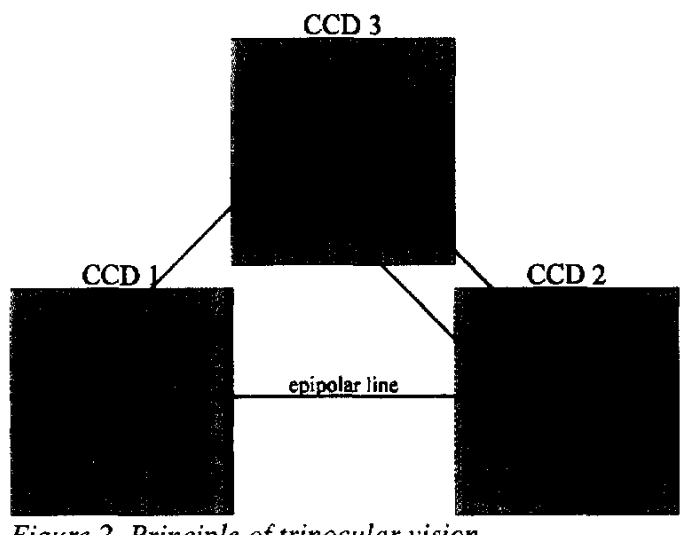

Figure 2. Principle of trinocular vision

Trying to find in CCD 2 or CCD 3 the corresponding feature point of $P_{1}$ in CCD 1 by using binocular vision does not give a unique solution. However, by using three cameras, in most cases a unique solution is found. Once a feature point in CCD 2 is selected $\left(\mathrm{P}_{2}\right.$ or $\left.\mathrm{P}_{3}\right)$, it is known that the corresponding feature point in $\mathrm{CCD} 3$ must lie on the epipolar line in CCD 3. But, it is also known that the feature point must lie on the epipolar line determined by the feature point from CCD 1 . So, the correct match must have a feature point at the intersection of the epipolar lines, only $\mathrm{P}_{4}$ fulfils this condition. Ambiguous solutions are found when there are two or more feature points, that lie at the intersection of epipolar lines.

Trinocular vision has a number of advantages over binocular vision, i.e. (Shen [8]):

- All binocular vision systems match the stereo pair images by the similarity of images. However, the system tries to find the 3-D data from the difference between them.

A trinocular system also tries to find the 3-D data from the difference between images, but the matching is done by the epipolar constraint rather than by the similarity between images.

- The precision of a binocular stereo vision system depends on the distance between cameras. When the distance between cameras is increased to improve the precision, the similarity hypothesis between the images will be more violated and it will be more difficult to match the images by similarity.

The precision of a trinocular system also depends on the distance between the cameras, but correspondence can always be found by the epipolar constraint.

- Almost all binocular vision systems match the feature pixels of feature primitives. But when the direction of a primitive composes a small angle with the epipolar line, the precision of the system will be bad.

In trinocular systems, the direction of the primitives is not necessarily used. Correspondence is found at the intersection of epipolar lines.

Altogether, the best method for the relief meter is trinocular vision. It is important to obtain accurate results. This weighs heavier than the extra costs and computational complexity connected to such a system.

\section{MATCHING CRITERION}

Due to various types of errors, there is still a chance that three feature points satisfying precisely the epipolar constraints, cannot be found. Therefore, we need a criterion to select corresponding points. Basically two approaches exist. In the first approach, only feature points that exactly fulfil the epipolar constraint are considered. By doing this, the errors induced by camera calibration, quantization effects and noise are ignored. On the other hand, it is easy to reconstruct the point in the scene: all three lines of sight of the feature points have an intersection in space. The second approach selects the feature point "closest" to the intersection of the epipolar lines in the third image as the best point. As Figure 3 shows this can easily lead to erroneous solutions.

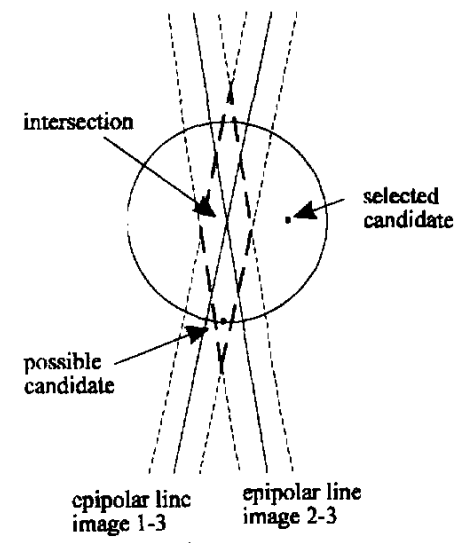

Figure 3. Erroneous selection

In Figure 3, epipolar line image $\mathrm{i}-\mathrm{j}$ is defined as the epipolar line in image $j$, belonging to a feature point in image i.

The best candidate is expected to lie on the intersection of both the epipolar lines $1-3$ and $2-3$. If this point is not a feature point, the feature point "closest" to the intersection point is accepted. The further a feature point deviates from the epipolar line, the smaller the chance that the point is a good candidate. The dotted 
lines in Figure 3 bound the maximum allowed deviation. Feature points outside these bounds are expected not to be a good match, so all feature points within the dotted diamond are possible candidates. Therefore, it is not correct to state that the best feature point is expected to lie on the intersection of the epipolar lines, and the further away from the intersection, the smaller the chance that the point is a good candidate. By doing so, the candidate that would be selected in Figure 3 is not a candidate at all, although its distance to the intersection is smaller than that of the only possible candidate.

By allowing a (small) deviation of the epipolar constraint in the third image, the reconstruction of the scene point has become more difficult. Therefore, the third image is only used to identify the correct match (and is not used to improve the accuracy of the reconstruction of scene points). The point in the scene is reconstructed using only the first two images. The lines of sight of the two feature points in these images have an intersection in space.

Because criteria based directly on the epipolar lines do not seem to function very well, we decided to use a different approach to select matching candidates. The feature point in the third image is expected to lie on the intersection of epipolar lines in the third image, so the three corresponding lines of sight are expected to have an intersection in space. If there is no feature point at the intersection of the epipolar lines, the three lines do not have an intersection in space. In order to select the best point in space corresponding to the three lines of sight, a penalty is given to a deviation of these lines of sight. In terms of estimation theory, this directly determines the object function to be minimised (Figure 4).

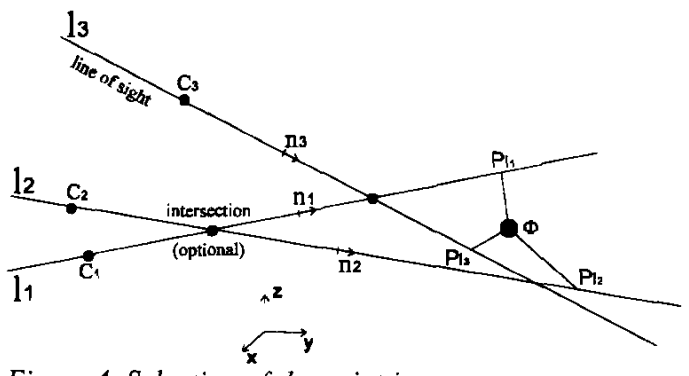

Figure 4. Selection of the point in space

The starting point is that we have three feature points, one in each image. These points determine three lines of sight $\left(l_{1}, l_{2}, l_{3}\right)$. We then want to determine:

- the likelihood that the three selected feature points are corresponding feature points,

- the point in space that is most likely the imaged scene point.

For this goal, we determine the following object function:

$$
J=\left\|\vec{P}_{l_{1}}-\vec{\phi}\right\|^{2}+\left\|\vec{P}_{l_{2}}-\vec{\phi}\right\|^{2}+\left\|\vec{P}_{l_{3}}-\underline{\phi}\right\|^{2}
$$

This object function attaches a measure to any combination of three lines of sight, or to a combination of three feature points on the three CCD chips. The combination of feature points that has the smallest measure is selected as the best combination. Solving the correspondence problem means continuously selecting candidates so that this object function is minimised.

When we define $\underline{\vec{P}}_{l_{1}}=\underline{\vec{C}}_{1}+\underline{\vec{n}}_{1} a, \underline{\vec{P}}_{l_{2}}=\underline{\vec{C}}_{2}+\underline{\vec{n}}_{2} b$ and $\underline{\vec{P}}_{i 3}=\underline{\vec{C}}_{3}+\underline{\vec{n}}_{3} c$, (where $\vec{C}_{i}$ is the position of the pinhole of camera $i$ and $\underline{n}_{i}$ is a vector in the direction of the line of sight of camera $i$ and $a, b$ and $c$ are running variables), the object function (equation (1)) is a function of six unknowns $(a, b, c, \vec{\phi})$. Minimising the object function means setting the six derivatives $\frac{\partial J}{\partial a}, \frac{\partial J}{\partial b}, \frac{\partial J}{\partial c}, \frac{\partial J}{\partial \underline{\phi}_{x}}, \frac{\partial J}{\partial \underline{\phi}_{y}}$ and $\frac{\partial J}{\partial \underline{\phi}_{z}}$ to zero. This yields six linear equations in the six unknowns. Eliminating $a, b$ and $c$ leads to the solution for $\vec{\phi}$.

Ayache [9] describes how the vectors $\underline{\vec{C}}_{i}, \underline{\vec{n}}_{i}$ and the epipolar lines are determined.

In order to be able to calculate the object function, we need to determine the three lines of sight from three points from the three images. These lines of sight are the inverse images of these three points. Before we can calculate the inverse images of these points, we must have a model of how these points are generated by the camera, or a camera model. A conventional pinhole camera model, as described in De Graaff [5] is used. In this model, a camera is modelled with thirteen parameters. However, we do not need to know every individual parameter. It suffices to know the perspective matrix $\mathbf{M}$ (Ballard [10]). The perspective matrix relates the scene points and image points in the following way:

$$
\left(\begin{array}{c}
\bar{P}^{*} \\
1
\end{array}\right)=\left(\begin{array}{c}
U \\
V \\
w \\
1
\end{array}\right)=\left[\begin{array}{cccc}
m_{11} & m_{12} & m_{13} & m_{14} \\
m_{21} & m_{22} & m_{23} & m_{24} \\
m_{31} & m_{32} & m_{33} & 1 \\
0 & 0 & 0 & 1
\end{array}\right]\left(\begin{array}{c}
\phi_{x} \\
\phi_{y} \\
\phi_{z} \\
1
\end{array}\right)
$$

where $(*)$ denotes homogeneous co-ordinates, thus:

$$
\vec{P}=\left(\begin{array}{l}
u \\
v
\end{array}\right)=\left(\begin{array}{l}
U / w \\
V / w
\end{array}\right)
$$

where $u$ and $v$ are the image co-ordinates of the image point $P$. 
As soon as this perspective matrix is known the camera has been calibrated.

\section{CAMERA CALIBRATION}

The camera calibration is done in two stages. In the lirst stage. the perspective matrix is determined by comparing the scene co-ordinates of a number of alibration objects with the corresponding image coordinates of these objects. Each time, a scene point $\overrightarrow{\mathcal{O}}=\left(\phi_{,} \phi_{,} \phi_{z}\right)^{T}$ is matched with its corresponding image point $\vec{P}=(u, v)^{T}$. this provides two linear equations on the eleven unknowns of $\mathbf{M}$ (Ballard [10]):

$m_{13} \phi_{1}-m_{12} \phi_{8}+m_{13} \phi_{2}+m_{1 \downarrow}-u \phi_{2} m_{31}-u \phi_{y} m_{32}-u \phi_{z} m_{33}=u$ $m_{2:} \phi_{1}+m_{22} \phi_{1}+m_{23} \phi_{i}+m_{24}-1 \phi_{x} m_{31}-1 \phi_{1} m_{32}-v \phi_{2} m_{33}=v$

With more than eleven equations, a least squares estimator is applied to estimate the perspective matrix. The second stage of the calibration is done in the field. Because we have determined an estimate of the three perspective matrices of the three cameras in the first calibration stage, we can automatically search for corresponding calibration objects (the objects have relatively large measurements, and there are approximately 15 objects in the images so that unique correspondence is easily obtained). Once a set of corresponding objects is found, the centres of gravity of these objects are calculated. We then know, that these centres of gravity correspond to one unique point in space. so the three lines of sight of these centres must have an intersection in space. This leads to three equations on the 33 unknowns (three perspective matrices) of the system.

\section{ERROR CAUSES}

An implicit assumption made in the use of the perspective matrix is that a point $P$ in the scene is projected onto a floating point value in the image. However, these values are mapped onto integer values due to the discrete nature of the imaging device. Similarly, the location of the projection of a scene point at image co-ordinates $(u, v)$ is not exact, but within $\left(u \pm^{1 / 2}, 1 \pm 1 / 2\right)$. Thus, in most cases it is impossible to exactly reconstruct this scene point. Several system parameters, such as the separation between the sensor elements, the sensor focal length, the sensor size and the sensor array dimensions influence the error made by reconstructing the scene point (McVey [11]).

\section{Camera set-up}

Unfortunately, one must compromise to meet the conflicting requirements of accurate feature matching and accurate range estimation. In order to avoid as much occlusion as possible, the products of baseline and focal length must be small. Accurate range estimation requires this product to be large (Rodriguez [12]). Alternatively, the range estimation accuracy can be improved by decreasing the sampling interval.

\section{Feature extraction errors}

In the current implementation, features are extracted by the Canny edge detector (Canny [13]). The detection of this operator consists of a magnitude-gradient determination of a Gaussian filtered image. Berzins [14] concluded that the errors induced by the edge extraction are small, provided that (amongst others) the size of the region is large compared to the operator size. This means, that smaller operator sizes give better localisation. On the other hand, an image filtered with a smaller operator size contains more edges and hence more feature points. This decreases the chance of finding a unique solution of the epipolar constraint.

For this reason, Grimson and others [15] proposed a coarse-to-fine strategy: In the first stage, images are filtered with a relatively large operator size. This gives a relatively small number of feature points, which makes the matching process easy. The result of this first stage is a sparse depth map. In consecutive stages, the images are filtered with a smaller operator size, thus producing more accurate results and a more dense depth map. The depth map from the previous stage is used for the verification of matching candidates.

\section{EXAMPLES}

In this section, we present a simple example of the results obtained with the described algorithm. First, the three perspective matrices were obtained by calibrating the cameras. In Figure 5, an example of the calibration scene is given. This scene is generated by a ray-tracer. Next, an artificial object is generated and imaged by the three cameras. In the three images, there are no occluding areas and no discontinuities. Feature points are the edges extracted by the Canny edge detector.

Figure 6 depicts the object and Figure 7 the object as it is observed by the first camera. Figure 8 depicts the extracted feature points. The final result of the reconstructed pyramid is presented in Figure 9.

\section{CONCLUSIONS}

The concept for the relief meter being developed, appears to function well, when used with the artificial images. The described matching criterion leads to high matching percentages, and accurate results. The percentage of mismatches is reduced to practically zero for the tested scenes.

Future work will involve evaluation of the algorithm with real agricultural scenes (soil images) and implementation of special hardware for fast execution of the algorithm. 


\section{REFERENCES}

[1] Kuipers. H., "A Relief Meter for Soil Cultivation Studies". Neth. J. Agric. Sci., 5, pp.255-262, 1957

[2] Römkens. M., Singaryar, D., Gantzer, C., "An Automated Non-contact Surface Profile Meter". Soil \& Tillage Research. 6. pp. 193-202,1986

[3] Römkens, M.. Wang. J.. Darden. R., "A laser Microreliefmeter", Transactions of the ASAE, vol. 31(2), pp. 408-413, March-April 1988

[4] Smith, G., "Stereo Reconstruction of Scene Depth". IEEE Comp.Soc. Conf.on Pattern Recognition. San Francisco, June 1985

[5] De Graaff, A.J., "Labconventies voor de parametrisatie van geometrische 3D-modellen". Internal Report No 92M016. University of Twente. Department of Electrotechnical Engineering. 1992

[6] Grimson, W., "From Images to Surfaces", MIT Press, Cambridge, MA, 1981

[7] Yachida, M., Kitamura, Y.. Kimachi, M. "Trinocular Vision: New Approach for Correspondence Problem", Proc. Int. Cont. Pattern Recognition, IEEE, Paris, France, Oct. 1986, pp. 1041-1044
[8] Shen, J., Castan, S.. Zhao. J., "A new passive measurement method by trinocular vision". Industrial Metrology 1.pp. 231-259. 1990

[9] Ayache, N., Lustman, F.. "Trinocular Stereo Vision for Robotics". IEEE Transactions on Pattern Analysis and Machine Intelligence, Vol. 13, No. 1. 1991

[10] Ballard, D., Brown. C., "Computer Vision". Prentice-Hall, Inc., 1982

[11] McVey, E., Lee, J., "Some Accuracy and Resolution Aspects of Computer Vision Distance Measurements", IEEE-PAMI, Vol. PAMI-4. No. 6 November 1982

[12] Rodríguez. J.. Aggerwal, J.. "Quantization Error in Stereo Imaging", IEEE. 1988

[13] Canny, J.. "Finding edges and lines in images". MIT internal report AI-TR-720, 1983

[14] Berzins, V., "Accuracy of Laplacian Edge Detectors", Computer Vision. Graphics, and Image Processing 27, pp. 195-210, 1984

[15] Grimson. W., "From Images to Surfaces". MIT Press. Cambridge, MA. 1981

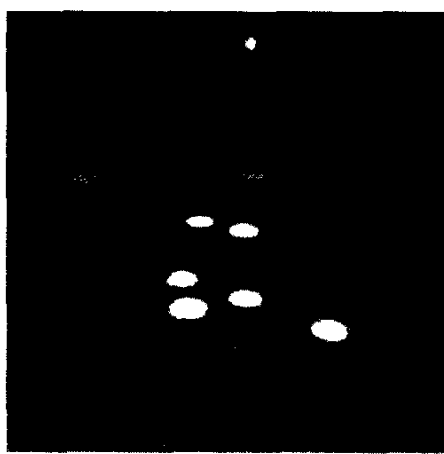

Figure 5. Calibration scene

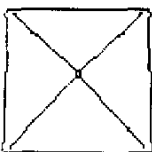

Figure 8. Extracted feature points

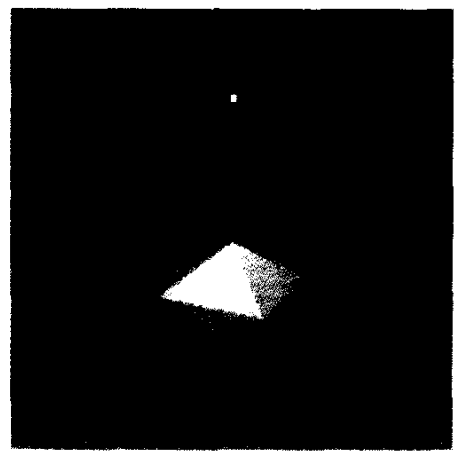

Figure 6. Object scene

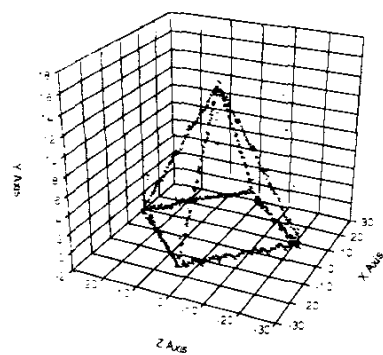

Figure 9. Reconstructed prramid

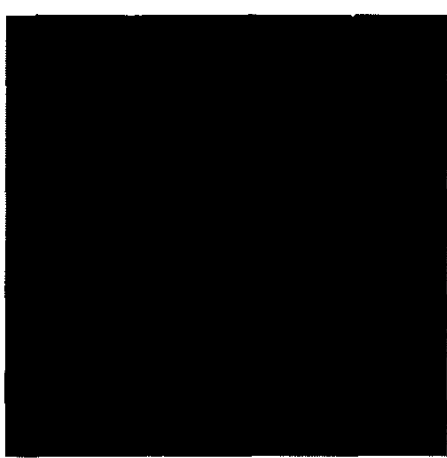

Figure 7. First object image 